\title{
DISCUSSION
}

\section{Dimensional analysis for geotechnical engineers}

\author{
R. BUTTERFIELD (1999), Géotechnique, 49, No. 3, 357-366.
}

B. V. S. Viswanadham, Indian Institute of Technology Bombay, D. König and H. L. Jessberger, Ruhr University

The discussers would like to commend the author for an interesting technical paper on dimensional analysis for geotechnical engineers. The ambiguities in arriving at the complete set of dimensionless groups (DGs) were clearly brought out by the author, but the discussers would like to $(a)$ emphasise the applications of dimensional analysis in physical modelling in geotechnical engineering, $(b)$ demonstrate the application of dimensional analysis in arriving at the assessment procedures, and $(c)$ supplement with another simple method for formulating DGs. The necessary and sufficient condition for establishing $N(=n-r)$ unambiguously depend upon the rank of the dimensional matrix formed by the indices of the fundamental dimensions of the set of variables rather than $m$ dimensions. The above condition is found to be in agreement with the one given by Douglas (1969): the number of DGs is equal to number of variables minus the number of independent indicial equations. For example, if we adopt $D=\{M, L, T\}$ for the given system having rank of the dimensional matrix $r=2$, the system will have only two independent indicial equations. Further, Douglas (1969) has given an alternative method for forming DGs based on the selection of $k$ fundamental variables out of $n$ variables to form $n-k$ DGs. The rank of the dimensional matrix formed by the set of variables $V=\{m o m, z, E I, h, \phi, \gamma, d\}$ is 2 . As $r=2$, it is required to select $k$ (in this case $k=2$ ) variables containing two fundamental dimensions force $[\mathrm{F}]$ and length [L]. Selecting $d$ and $\gamma$ and expressing the remaining $(n-k)$ variables in terms of fundamental dimensions $[\mathrm{L}]$ and $[\mathrm{F}]$, the following DGs can be obtained:

$$
\frac{m o m}{\gamma d^{4}}=f\left(\frac{z}{d}, \frac{E I}{\gamma d^{5}}, \frac{h}{d}, \phi\right)
$$

The relationship obtained is identical to one of the solutions given by the program code reported by the author. For a given set of variables defining the specific phenomenon, the program code provided by the author is helpful in arriving at the possible sets of dimensional groups. However, the correct sets of DGs are needed to be selected and have to be verified through experimental evidence.

One of the most important applications of DA for geotechnical engineers is the possibility of predicting full-scale behaviour from observations made on small-scale models tested under either $1 g$ or $n g$ conditions. The discussers have used dimensional analysis for understanding and interpreting the behaviour of numbers of geotechnical problems through centrifuge model tests. Many investigators (e.g. Ovesen, 1979; Cargill \& Ko, 1983; Goodings \& Santamarina, 1987; Jessberger \& Güttler, 1988; Arulanandan et al., 1988; Viswanadham, 1996), have used Buckingham's pi theorem for establishing the scaling relations between normal gravity, centrifuge models and prototypes. In many cases, DA alone is not sufficient to solve the problem. Quite often argument arises after performing the DA. For example, if grain size or characteristic macroscopic length (in the case of modelling contaminant transport) is introduced as an important parameter in any typical phenomena, at least one DG will be with grain size. The consequent effects of departure from complete similarity are believed to have a strong influence on independent DGs (such as $e, \phi$ ). In a model test, this DG will be satisfied, if $(a)$ the model has the scale 1:1 and prototype soil is used in the model, or $(b)$ the prototype soil is perfectly scaled down in the model (which seems to be impracticable). Because of this, some assumptions are indispensable in a problem where soil parameters/characteristics are predominant. These assumptions have to be examined by experimental procedures convincingly. Because of this, Ovesen (1979) has clearly stated that $(a)$ the complete set of similarity requirements must be established by means of DA, and $(b)$ the departure from complete similarity must be justified by means of experimental evidence.

Recently, while studying the integrity of clay liner systems, the discussers have demonstrated the relevance of dimensional analysis in studying the behaviour of clay liner systems in centrifuge. The resulting DGs can be combined to formulate a procedure for assessment of clay liners of landfills.

\section{INTEGRITY OF CLAY LINER OF LANDFILLS}

The clay liners of landfills are prone to cracking when subjected to excessive non-uniform settlements (Fig. 3). The crack depth $\left(d_{\mathrm{c}}\right)$ is a function of central settlement $(s)$, tensile strength of the liner material $\left(q_{\mathrm{d}}\right)$, overburden load $\left(\sigma_{0}\right)$, shear strength parameters $\left(c^{\prime}, \phi^{\prime}\right)$, outer fibre strain due to non-uniform settlements $\left(\varepsilon_{\mathrm{rf}}\right)$, tensile strain of liner material $\left(\varepsilon_{\mathrm{zq}}\right)$, unit weight of liner material $(\gamma)$ and thickness of liner material $(d)$. As the model involves only two fundamental dimensions, force $[\mathrm{F}]$ and length [L], it would be valid to form the dimensional matrix (Table 3) of the variables $\boldsymbol{V}=\left\{d_{\mathrm{c}}, s, q_{\mathrm{d}}, \sigma_{0}, c^{\prime}, \phi^{\prime}, \varepsilon_{\mathrm{rf}}\right.$, $\left.\varepsilon_{\mathrm{zq}}, \gamma, d\right\}, n=10$.

Since the second-order determinant at the right-hand side of the dimensional matrix in the last two columns is non-singular, the rank of the matrix is 2 (i.e. $r=2$ ). This is based on the hypothesis that a non-zero determinant of order $r$ occurs in the right-hand $r$ columns of the matrix. If the non-singular matrix is not found on the right-hand side of the dimensional matrix, it may be necessary to rearrange the variables after determination of the rank of the dimensional matrix. The rearrangement of variables in the columns will not change its rank (Langhaar, 1951).

Eight complete sets of DGs are possible for studying the integrity of the clay liner $(N=10-2)$. Writing the homogeneous linear algebraic equations whose coefficients are the numbers in the rows of the dimensional matrix (Table 3), we obtain:

$$
\begin{aligned}
& a_{3}+a_{4}+a_{5}+a_{9}=0 \\
& a_{1}+a_{2}-2 a_{3}-2 a_{4}-2 a_{5}-3 a_{9}+a_{10}=0
\end{aligned}
$$

By adopting the elementary elimination procedure and satisfying equations (14) and (15), the matrix of solutions can be obtained. The obtained solution is represented in the following equation, where $r^{\prime}, r^{\prime \prime}, r^{\prime \prime \prime}$ are dimensionless ratios:

$$
\pi_{1}=f\left(\pi_{2}, \pi_{3}, \pi_{4}, \pi_{5}, r^{\prime}, r^{\prime \prime}, r^{\prime \prime \prime}\right)
$$

The resulting eight DGs can be written as

$$
\frac{d_{\mathrm{c}}}{d}=f\left(\frac{s}{d}, \frac{q_{\mathrm{d}}}{\gamma d}, \frac{\sigma_{0}}{\gamma d}, \frac{c^{\prime}}{\gamma d}, \phi, \varepsilon_{\mathrm{rf}}, \varepsilon_{\mathrm{zq}}\right)
$$

By transformation of the above DGs the other pi terms such as $\left(d_{\mathrm{c}} \gamma / c^{\prime}\right),\left(\sigma_{0} / c^{\prime}\right)$ and $\left(\varepsilon_{\mathrm{rf}} / \varepsilon_{\mathrm{zq}}\right)$ are obtained and are used in developing the assessment procedure for clay liners subjected to non-uniform settlements.

The above example demonstrates the application of dimen- 


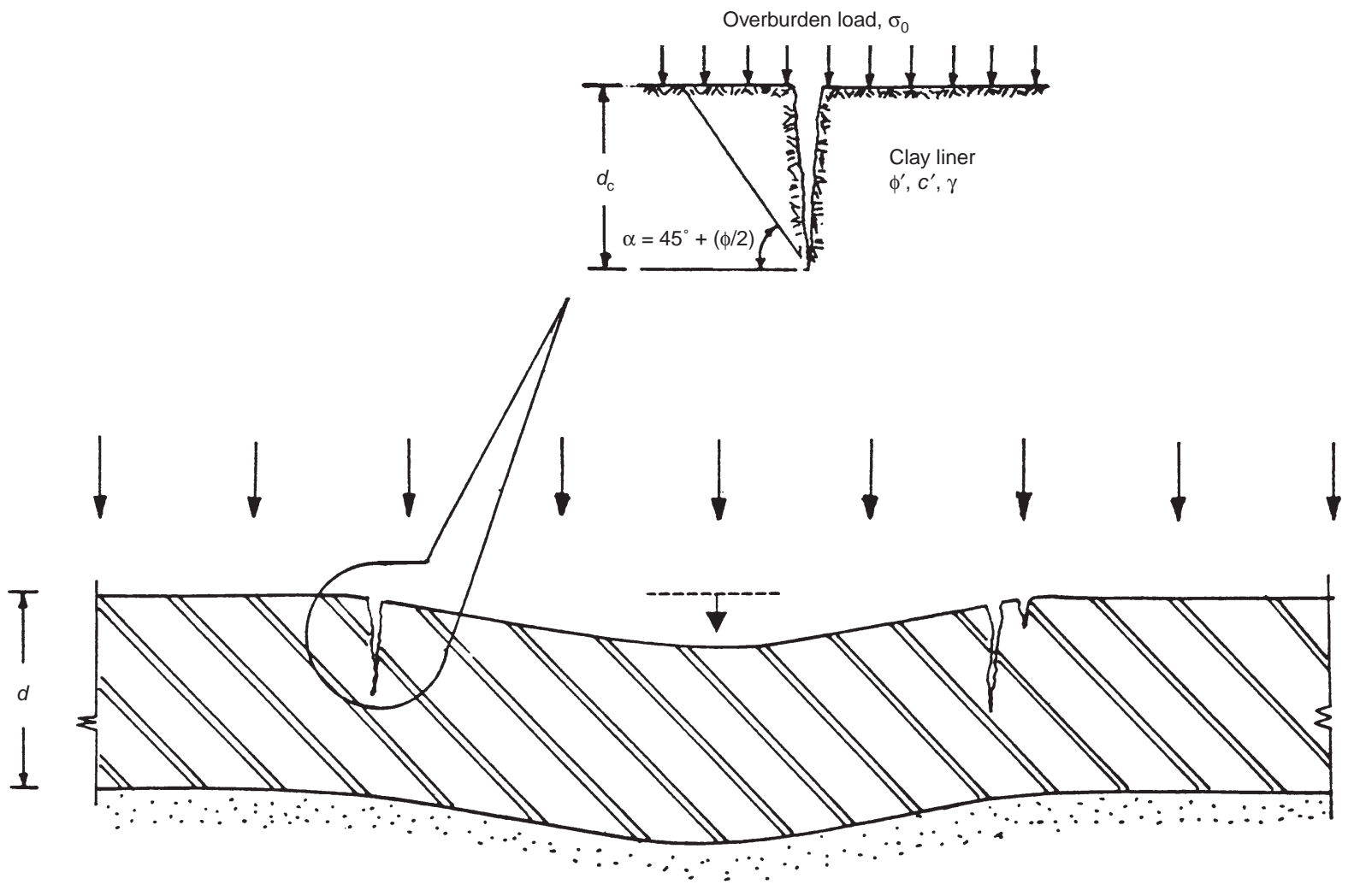

Fig. 3. Integrity of clay liner of landfills

Table 3. Dimensional matrix

\begin{tabular}{l|c|c|c|c|c|c|c|c|c|c}
\hline & $a_{1}$ & $a_{2}$ & $a_{3}$ & $a_{4}$ & $a_{5}$ & $a_{6}$ & $a_{7}$ & $a_{8}$ & $a_{9}$ & $a_{10}$ \\
& $d_{\mathrm{c}}$ & $s$ & $q_{\mathrm{d}}$ & $\sigma_{0}$ & $c^{\prime}$ & $\phi^{\prime}$ & $\varepsilon_{\mathrm{rf}}$ & $\varepsilon_{\mathrm{zq}}$ & $\gamma$ & $d$ \\
\hline $\mathrm{F}$ & 0 & 0 & 1 & 1 & 1 & 0 & 0 & 0 & 1 & 0 \\
$\mathrm{~L}$ & 1 & 1 & -2 & -2 & -2 & 0 & 0 & 0 & -3 & 1 \\
\hline
\end{tabular}

sional analysis to arrive at the assessment procedure for new phenomena. The complete similarity between DGs is achieved in centrifuge modelling as a result of testing of small-scale models in high gravity conditions compared with models tested at normal gravity conditions. This is especially possible when clay is used as the model material. This may be attributed to the reproduction of stress levels in the model that are identical to those of the prototype, and to stress-strain behaviour of model material that is similar to that of the prototype material. In the case of normal-gravity tests, it is necessary to reduce the effective cohesion, $c^{\prime}$, in order to satisfy the fifth pi term $\left[c^{\prime} / \gamma d\right]$, which will in turn influence the other DGs.

\section{Author's reply}

Viswanadham et al. correctly emphasise that is the rank $(r)$ of the 'indicial matrix' which determines the minimum number of dimensionless groups (DGs) to which any set of variables $(\boldsymbol{V})$, assumed to define some physical system adequately, can be reduced. However, as shown in my paper, the rank of the complete indicial matrix is governed by the dimensions of the components of $\boldsymbol{V}$, and is the same whatever set of 'primary or secondary' dimensions are used to describe them. The number of dimensions forming the set $\boldsymbol{D}_{\min }$ has to be equal to this rank in order for a valid DA to be achieved. Determination of the rank and, thereby, the relevant number of dimensions is therefore part of the problem: there are no generally applicable lists $\boldsymbol{D}$ of intrinsically 'fundamental' dimensions known a priori for all classes of DA problem.

Nor are there ' $k$ fundamental (isolated) variables' that can be selected other than by following the procedure described in the paper which defines a subset $\boldsymbol{R}$ of the $\boldsymbol{V}$ variables from which a smaller subset $\boldsymbol{Q}$ of repeated variables (in number equal to $r$ ) are to be selected. It is crucial that the components of $\boldsymbol{Q}$ cannot themselves form a DG. All the foregoing conditions are satisfied automatically by the code appended to the paper, which outputs all possible sets of DGs for a specific problem. It is important to emphasise that, contrary to the statement in the discussion, all such DGs are formally correct for any problem governed by the variables $\boldsymbol{V}$, although the list itself may be inadequate for its intended purpose. Different combinations of the DGs may be more convenient for either experimental investigation or the derivation of empirical equations fitted to data but, formally, there must always be $r$ of them.

The 'clay liner' DA presented in the discussion illustrates a straightforward application of the foregoing methodology. The rank of the ten-variable indicial matrix is clearly 2 and (Table 3 ) there are only three, dimensionally distinct, non-zero types of variable involved (i.e. $\boldsymbol{R}$ has three components, say $\left(c^{\prime}, \gamma, d\right)$ ) from which a $\boldsymbol{Q}$ with two components has to be selected, say $(\gamma, d)$. The $(10-2)=8$ DGs are then simply formed, by inspection, from combinations of $(\gamma, d)$ with the other eight components of $\boldsymbol{V}:\left(\mathrm{d}_{\mathrm{c}} / \mathrm{d}, \mathrm{s} / \mathrm{d}, \mathrm{q}_{\mathrm{d}} / \gamma \mathrm{d}, \sigma / \gamma \mathrm{d}, \mathrm{c}^{\prime} / \gamma \mathrm{d}, \varepsilon_{\mathrm{rf}}, \varepsilon_{\mathrm{zq}}, \varphi^{\prime}\right)$. It is incorrect to reduce the number of DGs to seven by using the ratio $\left(\varepsilon_{\mathrm{rf}} / \varepsilon_{\mathrm{zq}}\right)$ without experimental support. This applies equally to all of the DGs: there are minimally eight of them.

The discussion also includes comment on the application of DA to centrifuge-model testing. Problems can hardly ever be solved by DA alone, but if model and prototype measurements do not agree owing to incorrect scaling of the soil in the model this is hardly a deficiency of DA. It is also worth noting that fully correct scaling of the soil for a centrifuge model requires more than its being 'perfectly scaled down'. The soil-centrifuge DA problem is analysed in detail in Butterfield (2000), wherein, for example, the following scaling requirements, in addition to 
accurate representation of soil structure, are established if precise modelling is to be achieved (i.e. Reynolds' and Froude number, as well as effective stresses, porewater pressures etc., are all to be correctly represented).

Bold symbols indicate ratios of quantities (model to prototype). Thus $\mathbf{d}=\left(d_{\mathrm{m}} / d_{\mathrm{p}}\right)$ is the ratio of representative soilparticle sizes, $\mathbf{s}$ the geometrical scale, $\mathbf{v}$ the relative fluid to particle velocity ratio, $\boldsymbol{\mu}$ the fluid viscosity ratio, $\mathbf{t}$ the consolidation-time ratio and $\rho$ the density ratio (which has to be identical for both solid and fluid components). If the centrifuge acceleration ratio $\mathbf{g}$ is such that $\rho \mathbf{g s}=1$, then we require:

(a) If $\mathbf{d}=\mathbf{s}$ (scaled soil-particles) and $\boldsymbol{\mu}=\mathbf{s} \sqrt{ } \rho$, then $\mathbf{v}=1$ / $\sqrt{ } \rho$ and $\mathbf{t}=\mathbf{s} \sqrt{ } \boldsymbol{\rho}$

(b) If $\mathbf{d}=\rho=1$ (prototype particles) and $\boldsymbol{\mu}=\sqrt{ } 1 / \mathbf{s}$, then $\mathbf{v}=$ $\sqrt{ }(1 / \mathbf{s})$ and $\mathbf{t}=\mathbf{s}^{3 / 2}$

(c) If $\boldsymbol{\mu}=1$ (prototype fluid) and $\mathbf{d}^{3}=\mathbf{s}$, then $\mathbf{v}^{3}=\sqrt{ }(1 / \mathbf{s})$ and $\mathbf{t}=\mathbf{s}^{4 / 3}$

Even so, it is only in the first of these that particle spatial accelerations are modelled correctly. If prototype particles and prototype pore-fluid are used then neither the Reynolds' nor the Froude number will be correct, and the fluid velocity will be so large as to violate Darcy's law in many cases. That is:

(d) If $\mathbf{d}=\boldsymbol{\mu}=\boldsymbol{\rho}=1$ (prototype soil), then $\mathbf{v}=1 / \mathbf{s}$ and $\mathbf{t}=\mathbf{s}^{2}$
To model surface tension correctly, its ratio $\omega$ must equal $\mathbf{d}$ in all cases.

\section{REFERENCES}

Arulanandan, K., Thompson, P. Y., Kutter, B. L., Meegoda, N. J., Muraleetharan, K. K. \& Yogachandran, C. (1988). Centrifuge modelling of transport processes for pollutants in soils. J. Geotech. Engng Div., ASCE 114, No. 2, 185-205.

Butterfield, R. (2000). Scale modelling in a geotechnical centrifuge. Soils Found. 40, No. 6, 39-45.

Cargill, K. W. \& Ko, Hon-Yim (1983). Centrifuge modelling of transient water flow. J. Geotech. Engng Div., ASCE 109, No. 4, 536-555.

Douglas, J. F. (1969). An introduction to dimensional analysis for engineers. London: Pitman.

Goodings, D. J. \& Santamarina, J. C. (1987). Reinforced earth and adjacent soils: centrifuge modelling study. J. Geotech. Engng Div, ASCE 115, No. 7, 1021-1025.

Jessberger, H. L. \& Güttler, U. (1988). Geotechnical centrifuge Bochum: model tests in high acceleration fields. Geotechnik J., No. 2, 85-97.

Langhaar, H. (1951). Dimensional analysis and theory of models, John Wiley, New York.

Ovesen, N. K. (1979). The use of physical models in design. Proc. 7th Europ. Conf. Soil Mech. Found. Engng, Brighton 4, 319-323.

Viswanadham, B. V. S. (1996). Geosynthetic reinforced mineral sealing layers of landfills, Sr. No. 28, Dissertation of the Institute for Soil Mechanics and Foundation Engineering. Ruhr-University Bochum, Germany. 